Revista Calidad en la Educación Superior

Programa de Autoevaluación Académica

Universidad Estatal a Distancia, Costa Rica

ISSN 1659-4703

revistacalidad@uned.ac.cr

\title{
DESERCIÓN ESTUDIANTIL EN LA UNED: SEGUIMIENTO DE UNA COHORTE DE ESTUDIANTES DE PRIMER INGRESO
}

DESERTION IN UNED: TRACKING A COHORT OF FRESHMEN

Elisa Sánchez Godínez1, elsanchez@uned.ac.cr Escuela de Administración, Cátedra de Estadística Universidad Estatal a Distancia, Costa Rica

Volumen 6, número 1

Mayo 2015

Pp. 289 - 324

Recibido: 20 de agosto, 2014

Aprobado: 15 de abril, 2015

${ }^{1}$ Elisa Sánchez Godínez. Licenciada en Estadística, actualmente labora como encargada de la Cátedra de Estadística, Escuela de Administración. Universidad Estatal a Distancia, Costa Rica. 


\title{
Resumen
}

La retención estudiantil es una meta que persiguen todas las instituciones de enseñanza de un país o de una nación en cualquiera de sus niveles, ya sea en educación primaria, secundaria o universitaria. Es por esta razón que orientan todos sus esfuerzos a lograr que el y la estudiante inicien y culminen con éxito su formación. El otro extremo de la retención es la deserción, que es el desgranamiento 0 abandono de los estudios reflejado en un grupo de estudiantes desde que ingresa a una carrera hasta que toma la difícil decisión de no continuar en ella. Este trabajo pretende abordar este tema en la Universidad Estatal a Distancia de Costa Rica en un periodo de quince años, para lograrlo se le da seguimiento a una cohorte de estudiantes que ingresó por primera vez en el año 1998. La técnica de recolección de datos se basa en los registros administrativos; para su análisis se utiliza la técnica estadística llamada tabla de mortalidad, la cual es aplicada a la población de estudiantes de la UNED en el periodo 1998-2012.

Palabras claves: deserción en la educación superior, tablas de mortalidad

\begin{abstract}
The student retention, is a goal that all the educational institutions want to achieve in any of their levels (primary, high school or university), regardless the nation or the country. Is for this reason that all their efforts are oriented to motivate all the students since the beginning to the end of the entire process with successful. The other side of the retention is the desertion. The desertion is the abandonment of any kind of education and it can be reflected in a group of students since the first of their studies. This work aims to approach the student desertion in the "Universidad Estatal a Distancia de Costa Rica (UNED)", in a period of fifteen years. For make this article, is necessary a cohort of students that they were in the UNED for the first time in 1998. The recollection of the data is based on administrative records and for their statistical analysis is going to use the mortality tables as a statistical technique, the same is applied to cohort the students of the UNED in the period 1998-2012.
\end{abstract}

Keywords: desertion, abandonment, mortalitytables

El abordaje del fenómeno de la deserción es usualmente realizado mediante el seguimiento de cohortes de estudiantes en un periodo de tiempo, a este tipo de estudios se le conoce como estudios de cohortes o longitudinales. El rastreo da inicio con un grupo de personas que ingresan a estudiar en un determinado año y 
se les monitorea por un periodo de tiempo establecido, para registrar cuántos abandonan sus estudios y en qué momento.

Los estudios sobre deserción son realizados con el propósito de conocer el porcentaje de deserción que presenta una población específica, las causas o motivos que llevan a cada individuo a abandonar su carrera y, en algunas ocasiones, los años de estudio potencialmente perdidos. Con esta información las autoridades en materia de educación pueden implementar acciones en beneficio de estas poblaciones, para lograr que se mantengan en el sistema y culminen sus estudios con éxito.

Ninguna institución involucrada con educación escapa a este fenómeno, ya que se presenta en educación primaria, secundaria y universitaria. Según el Cuarto Informe Estado de la Educación, la deserción en secundaria pasó de 10,2\% en el año 2010 a 10,7\% en el año 2012. Señala el informe que en sétimo año pasó de 16,5\% a 16,7\% en 2010 y 2012, respectivamente (Estado de la Nación, 2013).

En educación superior universitaria el Consejo Nacional de Rectores (CONARE) ha sido el llamado a realizar estudios que involucran a las universidades estatales. Entre otras temáticas ha abordado el tema de la deserción, mediante el estudio de cohortes de estudiantes matriculados y matriculadas en estas universidades.

En una investigación sobre deserción y repitencia, en la que se da seguimiento por seis años a una cohorte de estudiantes que ingresaron en 1996 a las cuatro universidades estatales, se determinó un $68,6 \%$ de deserción en el año 2002 . Este estudio señala que alrededor del $50 \%$ de los y las estudiantes de la cohorte de 1996 desertó en el primer año en el que ingresó (Brenes, 2005).

De forma independiente, las universidades también han realizado algunos estudios. En su investigación sobre deserción Brenes (2006) señaló que la 
Universidad Nacional realizó en el año 1995 un seguimiento a la cohorte de estudiantes de 1990, y en 1999 la Universidad de Costa Rica analizó la cohorte de los y las estudiantes de esa universidad, que ingresó también en 1990. En ese mismo estudio se calcula el porcentaje de deserción de las cuatro universidades estatales, determinando que la UNED arroja porcentajes altos de deserción en sus carreras, todos superiores al $60 \%$. Brenes también señaló que en los dos primeros años se retiró alrededor del $70 \%$ de los y las estudiantes de esta universidad.

Específicamente, en la UNED en el año 1979 se dio el primer intento por contar con una cifra sobre deserción, para ese entonces el dato alcanzó un 35\%, mientras que en el II semestre de 1984 llegó a un 39,3\% y en el I semestre de 1985 a un $41,7 \%$ (Castillo, 2008).

A la fecha de esta publicación se han realizado estudios institucionales más recientes sobre este tema, enfocados en distintas ópticas, desde la descriptiva hasta la inferencial. Se han aplicado técnicas estadísticas que han permitido conocer el comportamiento de la deserción y contar con insumos para tomar decisiones. Estos estudios son mencionados más adelante en la sección de Investigaciones institucionales.

Recientemente, la UNED en su Reglamento General Estudiantil (2012b) define el periodo académico de referencia para clasificar a un estudiante como desertor. De manera que un estudiante que no matricule asignaturas durante cinco cuatrimestres consecutivos será clasificado como desertor. Con este marco de referencia cabe preguntarse de manera general ¿cuál es el comportamiento de la deserción estudiantil en la Universidad Estatal a Distancia bajo esta definición?, y en un sentido más específico ¿cuáles son los periodos académicos de mayor deserción? También surge la pregunta sobre ¿cuál ha sido la dinámica en el comportamiento de los y las estudiantes que ingresan en un año determinado, con relación a su permanencia o no en el sistema a distancia? 
Esta investigación pretende contestar estas interrogantes, para lo cual se utilizan registros administrativos de la matrícula de estudiantes de la UNED en el periodo 1998-2012. El universo de estudio considera la matrícula ordinaria en los programas de grado de las cuatro escuelas de esta institución.

Se hace uso de la base de datos contenida en el Sistema de Administración de Estudiantes más conocido como SAE. Para el estudio de los datos se aplicó el análisis de tablas de mortalidad, el cual brinda información acerca de la mortalidad de una población de estudio. Esta técnica es utilizada con el propósito de determinar la proporción de estudiantes que dejan de estudiar al final de cada año, determinando de esta manera la dinámica de cada grupo con relación al tema de la deserción.

Tal como se mencionó anteriormente, el estudio del fenómeno de la deserción abarca temas como el cálculo del porcentaje de deserción, los motivos o las causas de esa deserción y los años de estudios potencialmente perdidos en la formación del o de la estudiante; sin embargo, en este trabajo se va a centrar la atención en identificar los periodos académicos de mayor deserción y en el análisis de sobrevivencia a través de los resultados de las tablas de mortalidad, ya que por falta de recursos no se va a contactar a los y las estudiantes para indagar sobre las causas de su deserción.

\section{La deserción}

El sueño de la mayoría de jóvenes costarricenses que está por concluir su educación secundaria es obtener un título universitario que los faculte para desempeñarse como profesionales en un campo laboral determinado. Una cantidad de ellos lograrán ingresar a un programa o carrera de educación superior una vez que obtengan su título de secundaria, no obstante muchos de ellos se enfrentarán en algún momento de su vida estudiantil ante la decisión de seguir o no seguir con sus estudios superiores. El mismo hecho de hacer un alto en el 
camino y sopesar una situación personal determinada es difícil, sobre todo si la situación que enfrenta puede truncar de forma parcial o total sus sueños de superación.

Si por alguna razón el estudiante toma la decisión de abandonar las materias matriculadas y, por ende, sus estudios en determinada carrera o programa, este estudiante no logrará obtener un título universitario en ese programa y se convertirá en un estudiante desertor.

Según los textos consultados, los diferentes investigadores definen la deserción en función de múltiples factores. Ericka Himmel, en su estudio realizado en el año 2002 sobre modelos de análisis de la deserción estudiantil en la educación superior, definió la deserción como «el abandono prematuro de un programa de estudios antes de alcanzar el título o grado, y considera un tiempo suficientemente largo como para descartar la posibilidad de reincorporarse» (p. 94).

Además, Himmel (2002) señaló que existen dos tipos de deserciones: «La voluntaria y la involuntaria» (p.95). La primera apunta a una decisión tomada por el o la estudiante y la segunda a una decisión de la institución de educación, basada en sus reglamentos, tales como rendimiento académico bajo o por aspectos disciplinarios.

Osorio y Jaramillo (1999) definieron el abandono dependiendo del momento temporal en el que se dé, según los autores, puede existir un abandono parcial, que es cuando el o la estudiante suspende sus estudios por un motivo específico, pero los retoma en algún momento, y un abandono total, que es cuando el o la estudiante abandona sus estudios y no regresa. El abandono total es considerado por algunos autores como una deserción del sistema educativo. 
Montes, Almonacid, Gómez, Suluaga y Tamayo (2010) consideraron que la deserción «puede ser un concepto ambiguo, ya que puede tomar una multiplicidad de funciones y maneras de ser entendido» (p.13).

Los mismos autores, citando a Vásquez et al. (2003), indicaron que estos investigadores separan la deserción en dos tipos: la espacial y la temporal (precoz, temprana, tardía). La primera hace referencia al desplazamiento del o de la estudiante hacia otra carrera o centro de enseñanza o a su retiro del sistema. La segunda, por su parte, se refiere al abandono de la carrera en tres momentos: después de ser admitido o admitida, pero antes de que inicien las lecciones; durante los cuatro periodos académicos iniciales; o incluso posterior al quinto periodo académico (Montes et al, 2010).

La siguiente figura resume los diferentes conceptos utilizados para estudiar la deserción, tomando en consideración el tema espacial.

Figura 1. Niveles de estudio del fenómeno de la deserción

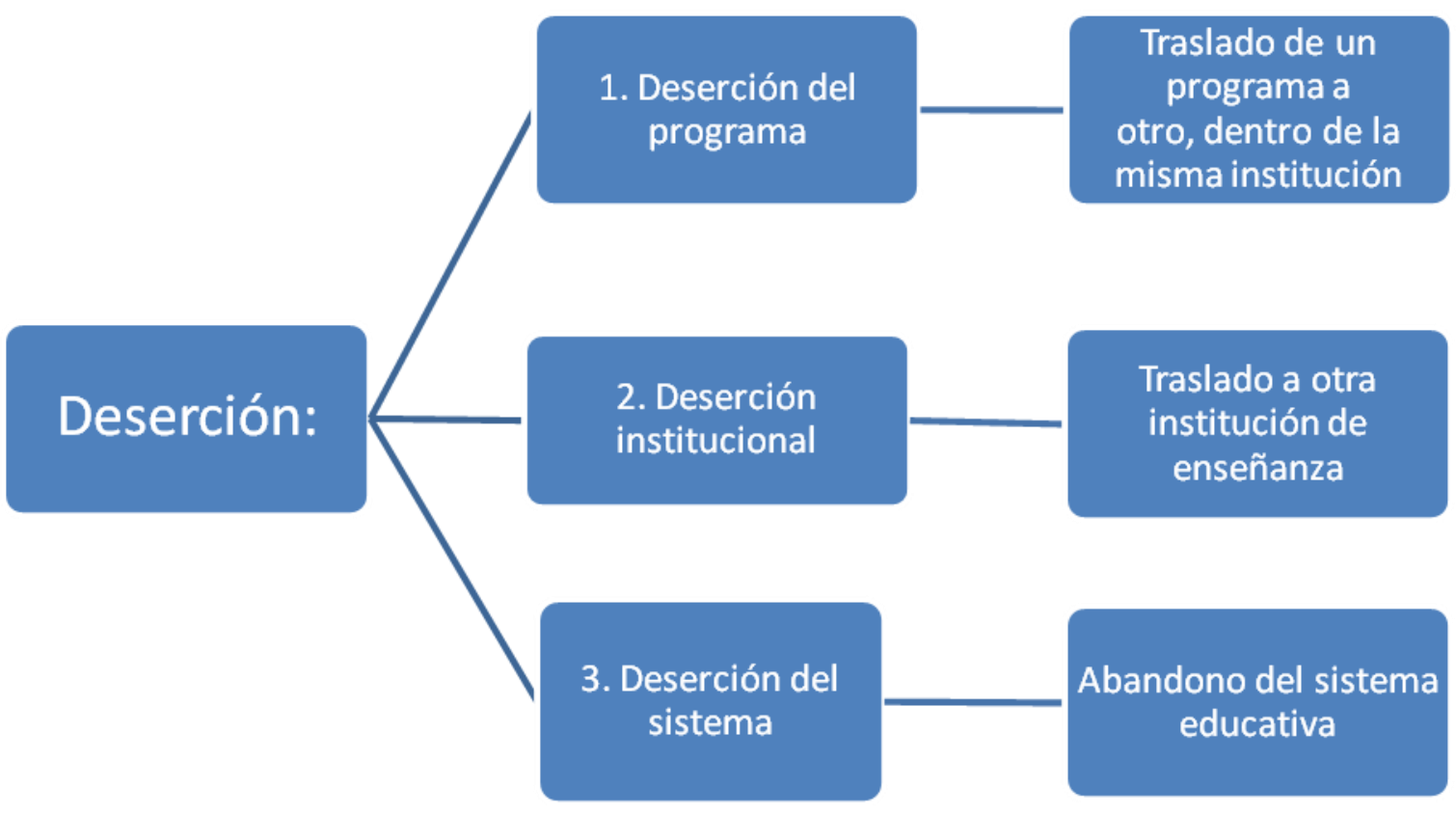

Fuente: Elaboración propia 
Desde un punto de vista más general, los dos primeros niveles de la figura 1 no deberían ser considerados como deserciones, debido a que el alumno o la alumna se mantiene estudiando en otro programa o en otra institución, mientras que el tercer nivel se refiere a un desertor o una desertora que no presenta ninguna posibilidad de retorno.

Según Himmel (2002), citando a Braxton et al. (1997), se han identificado cinco enfoques en el estudio del fenómeno de la deserción: los psicológicos, económicos, sociológicos, organizacionales y de interacciones.

El primero apunta a la personalidad del individuo, a sus atributos y actitudes; el segundo señala la capacidad o incapacidad de solventar los gastos asociados a los estudios; el tercero indica una influencia externa que dificulta su integración en el entorno estudiantil, tales como el medio familiar y la sociedad; el enfoque organizacional se dirige más hacia características institucionales, como los servicios que se brindan, la calidad de la docencia, la expectativa que tiene el individuo en el aula, ayudas y acompañamientos, material de apoyo, entre otros; y por último, la interacción es percibida como la integración y adaptación del o de la estudiante al sistema de educación (Himmel, 2002).

Estos enfoques pueden clasificarse en dos grupos si se toma como referencia la institución de educación superior. De esta manera, se pueden organizar en externos (los tres primeros) e internos (los dos últimos), con el propósito de identificar aquellos en los que la institución puede tener algún tipo de control y sobre los cuales puede orientar sus investigaciones (Himmel, 2002).

El fenómeno de la deserción es estudiado tanto en instituciones de educación superior presencial como a distancia. Algunos estudiosos del tema, como Chávez, Panchi y Montoya (2007), han señalado que el abandono de los estudios en la educación a distancia, para el caso de México, está fundamentado en causas 
específicas propias del sistema a distancia, en el que los y las estudiantes requieren motivación, disciplina y habilidades autodidactas. En su estudio los autores aplicaron cuestionarios y entrevistas a alumnos y alumnas, personas egresadas sin graduar y graduadas, docentes y coordinadores y coordinadoras de dos programas de maestría de la Escuela Superior de Comercio y Administración del Instituto Politécnico Nacional (IPN), en el periodo 1999-2003. Los puntos expuestos por las personas entrevistadas como importantes para bajar las tasas de deserción pueden clasificarse en tres grandes categorías: las características del o de la estudiante, la interacción con la persona tutora o asesora y los aspectos institucionales.

Kempfer (2001) realizó una revisión de 234 estudios de investigación sobre el tema de la deserción en la educación a distancia y señaló que el proceso de admisión, la calidad de los apoyos de aprendizaje y la interacción entre el tutor y el estudiante son tres elementos importantes para garantizar el éxito o el fracaso del estudiante.

\section{Características de la Universidad Estatal a Distancia de Costa Rica y sus esfuerzos por estudiar el tema de la deserción}

Hasta el año 2008 Costa Rica contaba con cuatro universidades públicas con más de treinta años de consolidación y con cincuenta universidades privadas que han surgido desde 1980 (CONARE-OPES, s.f.). Más recientemente, se creó la Universidad Técnica Nacional, según la ley N. ${ }^{\circ} 8638$ del 4 de junio de 2008. De todas ellas es la UNED la que utiliza la modalidad a distancia en el proceso de enseñanza-aprendizaje. En este modelo la población estudiantil organiza su tiempo de estudio dependiendo de sus responsabilidades y tiempo, no requiere que se presente a recibir clases en un aula, más bien su formación se realiza utilizando las Tecnologías de Información y Comunicación (TIC) como herramienta de aprendizaje a distancia. 
En 1977 la UNED aceptó el reto en su misión de ofrecer educación superior a todos los sectores de la población, particularmente a aquellos grupos que por su condición necesitan oportunidades educativas que les permitan insertarse de forma real y equitativa en la sociedad costarricense.

A diferencia de las otras instituciones de educación superior pública, la UNED no realiza examen de admisión. El o la estudiante efectúa directamente sus trámites de matrícula en dos modalidades, mediante la web o de forma presencial, sin embargo las personas que ingresan por primera vez deben realizar su matrícula de forma presencial. En esta modalidad el alumno y la alumna organizan la matrícula de las materias como mejor les convenga, avanzando en su programa de acuerdo a sus posibilidades.

Como parte de los apoyos facilitados a los y las estudiantes en su proceso de aprendizaje, la UNED brinda acceso a equipo de cómputo, capacitación en el uso de plataformas de aprendizaje, acceso a laboratorios de química y física, la producción de material de apoyo escrito y audiovisual, tutorías, becas, entre otros. Esta institución ofrece los servicios administrativos y docentes mediante los centros universitarios. Algunos de estos servicios no requieren que el o la estudiante se apersone al centro universitario para tramitarlos, sino que pueden acceder a la web de la institución.

En la actualidad existen 34 centros universitarios con instalaciones físicas distribuidos en todo el territorio nacional, además de tres subsedes. La mayoría de estas casas de enseñanza cuenta con laboratorios de cómputo con acceso a Internet, con el propósito de que la población estudiantil pueda acceder al material de apoyo y a las plataformas de aprendizaje en línea. Por el tipo de modalidad la población estudiantil debe tener conocimientos básicos de algunas herramientas de computación, o en caso contrario capacitarse en TIC. 
En cada periodo académico el alumno y la alumna reciben tutorías en los centros universitarios, con el propósito de evacuar dudas sobre temas de examen o sobre aspectos de alguna tarea o trabajo. Además de las tutorías las y los estudiantes cuentan con otras opciones como las llamadas telefónicas, los correos electrónicos o los foros en las plataformas de aprendizaje en línea.

Una diferencia importante de la educación a distancia en relación con la educación presencial es que el o la estudiante pocas veces sabe quiénes son sus compañeros y compañeras de curso, en ocasiones los conoce hasta el momento en el que se apersona al centro universitario a realizar un examen. Es por eso que la interacción con sus homólogos y homólogas muchas veces es escasa, lo que dificulta su adaptación al sistema.

La mayoría de estudiantes que escoge la modalidad a distancia lo hace porque reúne una serie de características que le impiden asistir presencialmente a clases. No obstante, al estar matriculados y matriculadas en un programa a distancia asumen un conjunto de desafíos que en muchas ocasiones les dificulta lograr la aprobación de materias. Cuando el estudiante reprueba uno, dos o más cursos puede entrar en una desmotivación que lo lleva con rapidez al abandono de los cursos y posteriormente a la deserción del programa y de la institución.

\section{Investigaciones institucionales}

La universidad ha realizado algunos esfuerzos por estudiar el tema de la deserción desde hace algún tiempo; las instancias que han apuntado en esta dirección son la Vicerrectoría de Planificación y la Vicerrectoría de Investigación, principalmente. Revisando algunos estudios y considerando los porcentajes que cada uno de ellos consigna en sus investigaciones, se puede decir que las cifras de deserción para la UNED oscilan entre el 35\% (1979) y poco más de 72\% (2011). 
Según Castillo (2008), en la UNED en el año 1979 se dio el primer intento por contar con una cifra sobre deserción, para ese entonces el dato alcanzó un $35 \%$, mientras que en el II semestre de 1984 llegó a un 39,3\% y en el I semestre de 1985 a un 41,7\%. Además mencionó dos estudios realizados en 1980 a petición del Rector de turno y el otro realizado en 1985, que es un estudio de opinión, para ambos estudios no presenta cifras.

Asimismo, Castillo mencionó que en una muestra realizada en 1987 en la misma universidad en 38 cursos impartidos en el II semestre de ese año se estableció un $54,6 \%$ de deserción.

Más recientemente, en el año 2000, el Centro de Investigación y Evaluación Institucional (CIEI) de la Vicerrectoría de Planificación realizó un estudio sobre el tema, en el cual determinó, mediante un análisis factorial, las materias con mayor grado de dificultad. El estudio concluye, entre otras cosas, que los porcentajes de deserción no han sufrido cambios de consideración en el periodo de estudio, indicando que el valor medio fue de $21 \%$ (Chaves, 2000).

En el 2001 la Vicerrectoría de Planificación realizó una investigación en la que se analizaron 234 estudios relacionados con la deserción en educación a distancia. Mediante ese estudio se concluyó que «hay tres elementos para determinar el éxito o fracaso individual. Ellos son: el proceso de admisión, la calidad de las relaciones entre el tutor y el estudiante y la calidad de los materiales de instrucción» (Kempfer, 2001, p.2).

Los resultados obtenidos por Castillo (2008) en su estudio sobre tasas de deserción en la UNED muestran que un $35 \%$ ha abandonado sus estudios en el primer semestre del año, al completar el año se acumula el $50 \%$ y posterior a ese periodo es menos acelerada esa acumulación, colocándose en $63 \%$ después de los 42 meses. 
En el 2009 se estudiaron las tasas de deserción en el Sistema de Estudios de Posgrado, concluyendo que de 127 estudiantes entrevistados el 19\% demostró intención de abandonar los estudios. De ellos $11 \%$ no terminó los cursos del programa, un $7 \%$ no presentó el examen de candidatura y un $1 \%$ presentó el examen, pero no la tesis (Barrientos y Umaña, 2009).

La Vicerrectoría de Investigación también ha realizado estudios sobre deserción, entre ellos el de Umaña (2011), que le dio seguimiento a una cohorte de estudiantes de primer ingreso en el año 2000 , concluyendo que $28,7 \%$ abandonó la UNED para estudiar en otra universidad y $43,6 \%$ la abandonó y no continuó estudiando.

Por su parte, la Vicerrectoría Académica, en el año 2004, trabajó en una propuesta contextual, teórica y metodológica para el abordaje de la deserción de los y las estudiantes en la UNED, en la cual se plantea una clasificación de ocho categorías de estudiantes de la universidad y señala que la deserción se debe abordar tomando como referencia a los que abandonan la universidad, los que aprueban algunos cursos (uso parcial) y se van, los que interrumpen estudios y los que reorientan sus estudios en otra universidad (Umaña, 2004).

\section{Rendimiento académico reportado en las materias impartidas en la UNED}

En los párrafos anteriores se hace mención a los porcentajes de deserción de los y las estudiantes en los diferentes periodos estudiados, sin embargo, no hay que olvidar que todo estudiante se enfrenta a una situación previa que se presenta cuando pierde las asignaturas o las abandona por diferentes motivos.

Una consulta realizada a las estadísticas sobre rendimiento de las materias impartidas en la UNED muestra que en el primer cuatrimestre del año 2000 se tenía un porcentaje de retiro de las asignaturas de 22,9\%, manteniéndose alrededor del $20 \%$ hasta el II cuatrimestre de 2005 , luego bajó a $14,4 \%$ en el III 
cuatrimestre de ese año y se mantuvo alrededor del $14,5 \%$ hasta final del periodo 2012 (cuadro 1).

Si bien es cierto que el rendimiento de los cursos no es lo mismo que la deserción de la carrera, lo cierto es que el bajo rendimiento en los cursos lleva a una posible deserción en un futuro cercano.

Según estas cifras se mantiene la aprobación de los cursos alrededor del $60 \%$ durante los quince años y la reprobación oscila entre 13,7\% y 18,5\% entre el 2000 y el 2005 , y entre $22,8 \%$ y $29,8 \%$ entre el 2005 y el 2012 , mientras que los retiros fueron desplazados a un tercer lugar por la reprobación, al pasar de cerca del $23 \%$ al $11,4 \%$ en 39 cuatrimestres.

El retiro de las asignaturas debe ser entendido como aquella situación en la que el o la estudiante deja de participar en las actividades programadas y no se presenta a realizar las evaluaciones de los aprendizajes, este puede ser justificado ante la Oficina de Registro o puede ser injustificado.

Cuadro 1. UNED. Rendimiento académico de las asignaturas matriculadas por los y las estudiantes, según cuatrimestre

\begin{tabular}{|c|c|c|c|c|}
\hline & & Porcentajes & & \\
\hline & efectiva & Aprobación & Reprobación & Retiros \\
\hline $2000-1$ & 32771 & 59,7 & 17,4 & 22,9 \\
\hline $2000-2$ & 31034 & 60,6 & 16,4 & 23,0 \\
\hline $2000-3$ & 27976 & 59,8 & 18,1 & 22,1 \\
\hline $2001-1$ & 35373 & 51,9 & 17,1 & 31,0 \\
\hline $2001-2$ & 32678 & 54,3 & 18,5 & 27,2 \\
\hline $2001-3$ & 29249 & 57,9 & 17,6 & 24,5 \\
\hline
\end{tabular}


Deserción estudiantil en la UNED: seguimiento de una cohorte de estudiantes de primer ingreso Elisa Sánchez Godínez

\begin{tabular}{|c|c|c|c|c|}
\hline $2002-1$ & 38724 & 58,0 & 15,9 & 26,1 \\
\hline $2002-2$ & 35488 & 62,1 & 15,7 & 22,2 \\
\hline $2002-3$ & 31839 & 62,2 & 16,3 & 21,5 \\
\hline $2003-1$ & 41816 & 61,7 & 16,0 & 22,3 \\
\hline $2003-2$ & 36163 & 64,8 & 13,7 & 21,5 \\
\hline $2003-3$ & 34231 & 61,4 & 18,3 & 20,3 \\
\hline 2004-1 & 39518 & 60,3 & 16,0 & 23,7 \\
\hline $2004-2$ & 37441 & 62,7 & 18,4 & 18,9 \\
\hline 2004-3 & 33657 & 61,3 & 18,6 & 20,1 \\
\hline $2005-1$ & 40705 & 61,5 & 15,8 & 22,7 \\
\hline $2005-2$ & 36567 & 63,0 & 15,4 & 21,6 \\
\hline $2005-3$ & 32543 & 62,8 & 22,8 & 14,4 \\
\hline $2006-1$ & 40450 & 57,9 & 26,3 & 15,8 \\
\hline $2006-2$ & 35706 & 60,7 & 25,3 & 14,1 \\
\hline $2006-3$ & 32730 & 60,1 & 25,1 & 14,8 \\
\hline $2007-1$ & 40208 & 57,9 & 26,6 & 15,4 \\
\hline $2007-2$ & 37223 & 58,0 & 27,3 & 14,8 \\
\hline $2007-3$ & 33190 & 57,7 & 28,3 & 14,0 \\
\hline $2008-1$ & 38956 & 56,5 & 26,2 & 17,3 \\
\hline $2008-2$ & 36586 & 55,6 & 29,0 & 15,4 \\
\hline $2008-3$ & 33379 & 56,2 & 29,8 & 14,0 \\
\hline $2009-1$ & 40004 & 56,2 & 28,0 & 15,8 \\
\hline $2009-2$ & 36757 & 59,2 & 26,7 & 14,1 \\
\hline $2009-3$ & 36102 & 60,9 & 26,3 & 12,9 \\
\hline $2010-1$ & 44896 & 54,1 & 29,7 & 16,2 \\
\hline $2010-2$ & 41238 & 58,0 & 27,8 & 14,2 \\
\hline $2010-3$ & 38180 & 57,1 & 28,9 & 14,1 \\
\hline $2011-1$ & 46560 & 54,4 & 29,5 & 16,0 \\
\hline $2011-2$ & 43390 & 58,2 & 27,9 & 13,8 \\
\hline $2011-3$ & 40084 & 57,0 & 29,2 & 13,7 \\
\hline $2012-1$ & 46928 & 57,8 & 27,2 & 15,1 \\
\hline $2012-2$ & 43682 & 60,9 & 27,4 & 11,8 \\
\hline
\end{tabular}




$\begin{array}{lllll}2012-3 & 41025 & 61,6 & 27,0 & 11,4\end{array}$

Fuente: UNED (2012a). Anuario estadístico. Centro de Investigación y Evaluación Institucional, Universidad Estatal a Distancia. San José, Costa Rica.

\section{Definición de deserción según el Reglamento General Estudiantil}

En la actualidad, según el Reglamento General Estudiantil (RGE) de la UNED (en su capítulo IV, artículo 4, inciso p), se define deserción como el «abandono de los estudios en la carrera en el que está empadronado cada estudiante, por cuatro o más periodos académicos consecutivos» (UNED, 2012b, p.9).

En el inciso u del mismo capítulo se define al estudiante inactivo que deserta como la "condición que cada estudiante adquiere cuando tiene cinco períodos académicos consecutivos o más que no matricula ninguna asignatura» (UNED, 2012b, p.9).

No obstante estas definiciones, hay que considerar que un o una estudiante pudo haber suspendido sus estudios temporalmente por un año o más, y regresar poco tiempo después, en este caso debería haberse clasificado con una condición de interrupción de estudios y no como desertor o desertora. Cabe mencionar que la suspensión temporal puede darse por la obtención de una beca, una situación familiar, una situación de trabajo, entre otros motivos.

Según algunas estadísticas extraídas de la base de datos institucional sobre graduados del año 2011, de un total de 2.252 graduados un $37,3 \%$ suspendió sus estudios en algún momento de su carrera. El promedio de años que estos 841 estudiantes suspendieron sus estudios fue de 5,3 años, con una desviación estándar de 6,8 años. 
Un $61,7 \%$ suspendió sus estudios por cuatro o menos periodos académicos, mientras que un $28,8 \%$ lo hizo por más de cuatro. Todos ellos retomaron sus estudios y lograron terminar su carrera. Si se le aplica a esta población la definición de deserción del RGE, se tendría que la cantidad de graduados y graduadas, correspondiente a este último porcentaje, sería contada como desertora sin serlo, sobreestimando de esta manera el porcentaje de deserción de la universidad.

La definición del RGE no hace distinción entre el abandono parcial y el total, tal como lo definieron Osorio y Jaramillo (1999), de manera que un abandono parcial puede estar siendo tomado como un abandono total, como es el caso mencionado anteriormente sobre los graduados y las graduadas. En ambas situaciones son diferentes las acciones que debe tomar la institución.

Recordemos que en la figura 1, mostrada previamente, se presentan tres niveles de deserción: la deserción del programa, la deserción de la institución y la deserción del sistema. En este último caso el o la estudiante no tiene opción de regresar ni a la misma institución de la cual desertó ni a otra dentro del mismo sistema de educación superior.

La definición empleada en el RGE pareciera circunscribir la deserción al primer nivel, considerando como desertores y desertoras a los y las estudiantes que se trasladan a otra carrera dentro de la misma institución. Esta situación es la realidad de la carrera, pero no de la universidad, dado que, a pesar de que el o la estudiante se mueve a otra carrera, todavía continúa en la misma institución de enseñanza.

Este trabajo centra la atención en la deserción institucional con el propósito de conocer su comportamiento, basado en el monitoreo de una cohorte de estudiantes de la UNED en un periodo de quince años. Esto quiere decir que no 
se estudian los casos de estudiantes que dejaron de matricular asignaturas en una carrera específica, dado que se han trasladado a otra carrera dentro de la misma institución.

\section{Población de estudio}

A diferencia de algunos estudios sobre este tema, en esta ocasión se trabajó con toda la población de estudiantes de primer ingreso del año 1998, a los cuales se les dio seguimiento de cuatrimestre a cuatrimestre usando la información sobre matrícula almacenada en el Sistema de Administración de Estudiantes, durante un periodo de quince años.

Se definió la población de estudio como todos los estudiantes de primer ingreso de la UNED que matricularon en cualquiera de los tres cuatrimestres del año 1998. Recordemos que en esta universidad ingresan estudiantes nuevos en cualquiera de los cuatrimestres del año.

Con el propósito de identificar si existen diferencias en el comportamiento de cada grupo de estudiantes se va a trabajar el grupo que ingresó en el año 1998 como si fueran tres cohortes independientes.

\section{Periodo de estudio}

Este periodo consta de los años comprendidos entre 1998 y 2012.

\section{Procedimiento metodológico}

Se consultaron documentos, libros y artículos relacionados con el tema de la deserción en instituciones presenciales y a distancia. Se consultó la base de datos del Sistema de Administración de Estudiantes para extraer datos sobre la matrícula ordinaria de primer ingreso, para el I cuatrimestre del año 1998. 
Se consultó esa misma base de datos para extraer la información de la matrícula total del II cuatrimestre de 1998 al III cuatrimestre de 2012, o sea todos los estudiantes que estuvieron activos en ese periodo de tiempo.

Se le dio seguimiento a los y las estudiantes de primer ingreso del año 1998, monitoreando su matrícula en los cuatrimestres subsiguientes a su primera matrícula, durante el periodo de estudio. Se identificaron los casos censurados (activos y graduados) con el número cero y los que presentan la ocurrencia del evento (deserción) con el número uno.

Los graduados son aquellos estudiantes que recibieron su título universitario una vez que lograron completar el plan de estudios y cumplieron con todos los requisitos para optar por este documento. Los estudiantes activos son los que matricularon regularmente las materias, indistintamente de que las hayan aprobado o reprobado.

Los desertores se definieron como los que durante cinco periodos consecutivos dejaron de matricular materias según el Reglamento General Estudiantil vigente a la fecha de elaboración de este trabajo.

Se aplica el análisis de tablas de mortalidad a la información extraída del sistema, relacionada con las tres cohortes de estudiantes de primer ingreso del año 1998, para determinar la función de riesgo, o sea la probabilidad de que el estudiante abandone sus estudios en el momento $t$, condicionado a que ha permanecido activo hasta ese momento t. De esta manera se puede determinar si la deserción ocurre y en qué momento ocurre (Ministerio de Educación Nacional, 2009). 


\section{Resultados}

Con el propósito de estudiar el fenómeno de la deserción se dio seguimiento en el tiempo a tres cohortes de estudiantes de primer ingreso de la Universidad Estatal a Distancia que matricularon sus primeros cursos en los cuatrimestres del año 1998. En la primera cohorte (I semestre 1998) se matricularon un total de 2782 nuevos estudiantes, mientras que en la segunda cohorte (II cuatrimestre) se contó con un total de 2233 jóvenes y adultos que ingresaron por primera vez a estudiar en la UNED. Por último, en el III cuatrimestre de 1998 se matricularon un total de 1641 estudiantes de primer ingreso, para un total de 6656 estudiantes de primer ingreso en todo el año 1998.

\section{Deserción del primero al segundo cuatrimestre}

El fenómeno de la deserción se presenta con mayor frecuencia al pasar del primero al segundo periodo académico, ya que el o la estudiante experimenta el ritmo académico y el nivel de exigencia de la carrera escogida. En el caso colombiano, un $37 \%$ de los y las estudiantes de una cohorte determinada abandonó sus estudios en el primer semestre de su ingreso (Ministerio de Educación Nacional, 2009).

Según el estudio realizado por Brenes (2006), los dos primeros años se retira alrededor del $70 \%$ de los y las estudiantes de la UNED.

Esta situación no es ajena a nuestro caso de estudio, los primeros datos señalan que es alto el porcentaje de los y las estudiantes que no presentan matrícula en el siguiente cuatrimestre a su ingreso. Para la cohorte 1998-1 de un total de 2782 nuevos estudiantes un $35,62 \%$ no logró matricular en el siguiente cuatrimestre, la segunda cohorte presentó un porcentaje levemente inferior en 1,54 puntos, en donde un $34,08 \%$ de los 2233 estudiantes no matricularon cursos en el siguiente periodo académico. La tercera cohorte se asocia con un $45,16 \%$ de los estudiantes sin matrícula en el siguiente cuatrimestre (cuadro 2). 


\section{Cuadro 2. UNED. Comportamiento de la matrícula de los y las estudiantes en el siguiente cuatrimestre a su ingreso (1998)}

\begin{tabular}{l|l|l|l}
\hline & $\mathbf{1 9 9 8 - 1}$ & $\mathbf{1 9 9 8 - 2}$ & $\mathbf{1 9 9 8 - 3}$ \\
\hline a) Estudiantes de primer ingreso & 2782 & 2233 & 1641 \\
\hline $\begin{array}{l}\text { b) Estudiantes que matricularon } \\
\text { en el siguiente periodo. }\end{array}$ & 1791 & 1472 & 900 \\
\hline $\begin{array}{l}\text { c) Estudiantes que no } \\
\text { matricularon en el siguiente } \\
\text { periodo. }\end{array}$ & 991 & 761 & 741 \\
$\begin{array}{l}\text { d) Porcentaje de estudiantes que } \\
\text { no matriculó en el siguiente } \\
\text { periodo. }\end{array}$ & 35,62 & 34,08 & 45,16 \\
\hline
\end{tabular}

Fuente: elaboración propia

\section{Períodos académicos de mayor deserción}

Además de conocer la magnitud del porcentaje de deserción en el segundo cuatrimestre de matrícula, un paso adicional es identificar en qué periodo académico se presenta este fenómeno con mayor frecuencia. La información analizada presenta un comportamiento similar al caso colombiano, en el que los porcentajes de deserción se presentan en los primeros tres periodos académicos, es más, en los dos primeros periodos se presenta más del $50 \%$ de la deserción (Ministerio de Educación Nacional, 2009).

En este caso, en la cohorte 1998-1 en los primeros dos cuatrimestres se presenta un $47,2 \%$ de la deserción (gráfico 1), la segunda cohorte muestra un porcentaje de $52,4 \%$ (gráfico 2) y la tercera un porcentaje de $54,8 \%$ (gráfico 3 ). 
Al acumular los primeros tres cuatrimestres se observa que el porcentaje de deserción se aproxima al $60 \%$. La primera cohorte muestra un $55,7 \%$ como porcentaje de deserción, la segunda un $60 \%$ y la tercera un $59,1 \%$.

Una cifra global que encierra las tres cohortes muestra que un $82,83 \%$ del total de estudiantes se identificó como desertor en algún momento del periodo de estudio al aplicar la definición consignada en el Reglamento General Estudiantil.

\section{Gráfico 1. UNED. Cuatrimestres con los mayores porcentajes de deserción} (primera cohorte 1998-1)

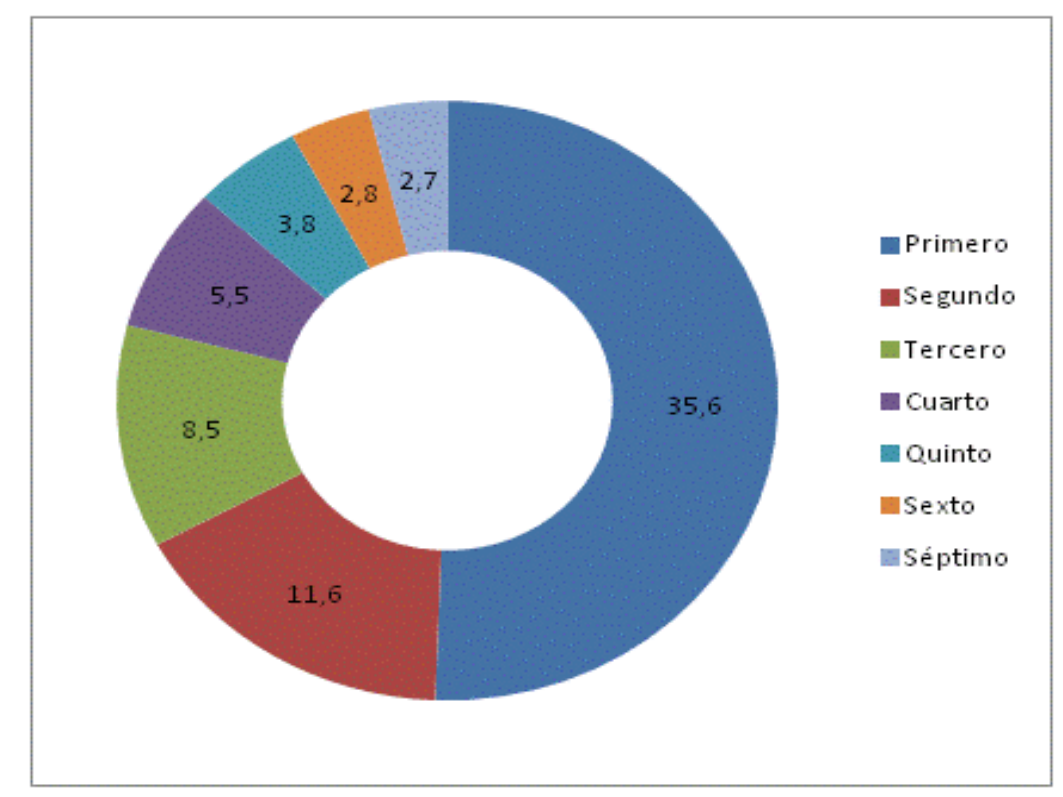

Fuente: En los cuatrimestres del octavo en adelante los porcentajes de deserción son inferiores a

$2 \%$. Elaboración propia 


\section{Gráfico 2. UNED. Cuatrimestres con los mayores porcentajes de deserción (segunda cohorte 1998-2)}

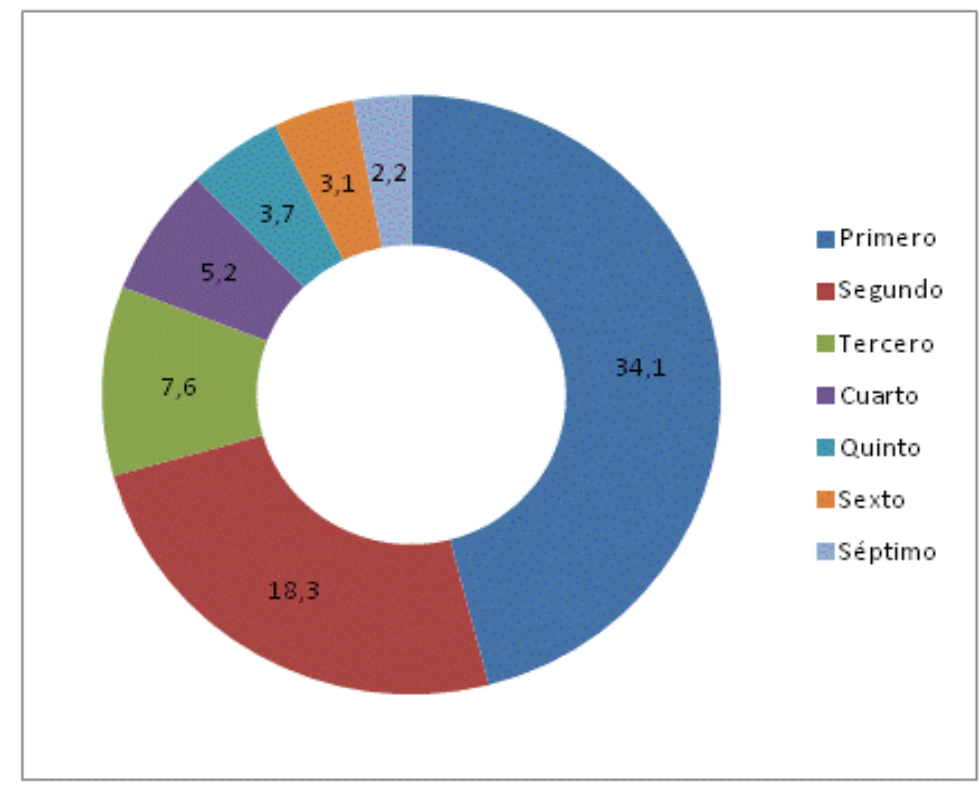

Fuente: En los cuatrimestres del octavo en adelante los porcentajes de deserción son inferiores a

$1,5 \%$. Elaboración propia

Gráfico 3. UNED. Cuatrimestres con los mayores porcentajes de deserción (tercera cohorte 1998-3)

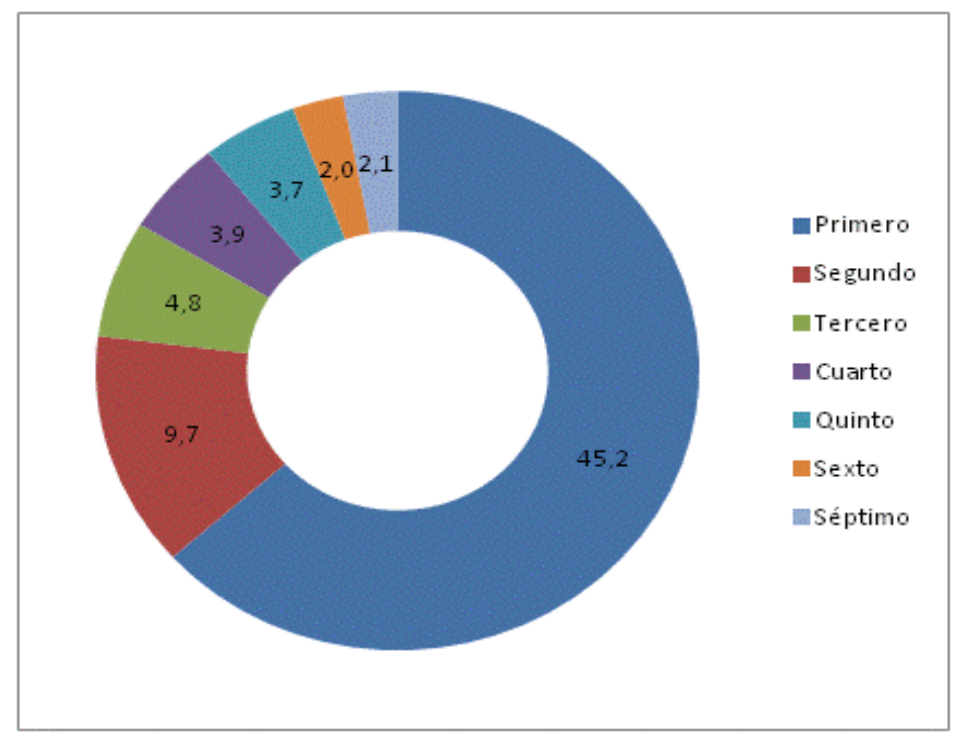

Fuente: En los cuatrimestres del octavo en adelante los porcentajes de deserción son inferiores a

1,5\%. Elaboración propia 


\section{Estudiantes activos y graduados al final del periodo de estudio}

Una vez que se ha conocido el comportamiento de la deserción de las tres cohortes estudiadas, cabe preguntarse qué porcentaje de los y las estudiantes logró titularse y qué porcentaje se ha mantenido activo. Sobre todo porque, mirando las cifras altas de deserción, queda un porcentaje inferior al $20 \%$ bajo el cual se pueden distribuir los porcentajes de graduados y estudiantes activos.

Del total de 6656 estudiantes de primer ingreso un 16,89\% (1124) obtuvo al menos un título con niveles de pregrado, grado o posgrado, 0,36\% (24 personas) había matriculado al menos una vez al año, pero sin obtener ningún título, esto quiere decir que en el periodo de estudio nunca obtuvieron una condición de desertores ni se graduaron, o sea que son estudiantes que se han mantenido en el sistema y están rezagados.

En el siguiente cuadro puede apreciarse el comportamiento de las tres cohortes, de acuerdo a los tres estados identificados.

Cuadro 3. UNED. Estudiantes de las tres cohortes estudiadas según
situación académica al finalizar el periodo de estudio (1998-2012)

\begin{tabular}{|l|l|l|l|}
\hline Estudiantes que & $1998-1$ & $1998-2$ & $1998-3$ \\
\hline a) obtuvieron un título universitario. & 496 & 340 & 288 \\
\hline $\begin{array}{l}\text { b) se mantienen activos, pero no } \\
\text { obtienen título universitario } \\
\text { (rezagados). }\end{array}$ & 13 & 2 & 9 \\
\hline c) desertaron. & 2273 & 1891 & 1344 \\
\hline Total & 2782 & 2233 & 1641 \\
\hline
\end{tabular}

Fuente: elaboración propia

\section{Tabla de mortalidad}

En este trabajo se utiliza la tabla de mortalidad como alternativa del análisis de duración de Kaplan-Meier por tratarse de una cantidad considerable de casos. En 
la tabla de mortalidad la primera columna se refiere a intervalos de tiempo de tres cuatrimestres cada uno, estos intervalos son interpretados como años de matrícula de cada grupo de estudiantes. El primer intervalo se refiere al primer año de matrícula, el segundo se asocia con el segundo año de matrícula y así sucesivamente hasta completar quince años de seguimiento.

La segunda columna indica la cantidad de estudiantes que inicia en cada intervalo de tiempo, la tercera muestra los casos censurados (graduados y los que no presentaron el evento de interés). La cuarta y quinta columna presentan cifras absolutas y relativas de la cantidad de estudiantes que abandona sus estudios. La sexta columna indica la proporción acumulada de estudiantes que se han mantenido en el sistema, lo que quiere decir que el complemento es la proporción de estudiantes desertores. Por último, la columna sétima es la función de riesgo o tasa de azar, la cual se define como la probabilidad de que un estudiante que se matricula en el intervalo $t$ se mantenga activo en el siguiente intervalo de tiempo lo suficientemente pequeño.

Los datos de la tabla de mortalidad señalan que después del primer año de matrícula de los y las estudiantes alrededor el $50 \%$ de ellos había dejado de matricular cursos, el $47,2 \%$ de los y las estudiantes de la primera cohorte había desertado, un $52,4 \%$ de la segunda y un $54,8 \%$ de la tercera.

Al concluir el segundo año de matrícula los porcentajes acumulados oscilaron entre el $65 \%$ y el $69 \%$, estos porcentajes se calculan restando a la unidad el valor relativo de la columna llamada Proporción acumulada que sobrevive al final del intervalo. Un $65 \%$ de estudiantes de la primera cohorte había desertado, un $69 \%$ de la segunda cohorte y un $67 \%$ de la cohorte 1998-3. Y para el tercer año de matrícula los porcentajes acumulados ya superaban el $70 \%$ (ver tabla de mortalidad). 
Hasta los siete años de matrícula el comportamiento de los porcentajes de deserción se mueven con rapidez al pasar de $0 \%$ a $85 \%$, después de este año se desaceleran y cambian más lentamente. En estos últimos ocho años los porcentajes apenas se mueven cinco puntos porcentuales al pasar de $85 \%$ a $90 \%$ en la primera y tercera cohortes, mientras que en la segunda cohorte se mueve seis puntos porcentuales al pasar de $87 \%$ a $93 \%$.

Al final de los quince años de seguimiento de cada cohorte las cifras de deserción acumuladas llegaron al $90 \%$ en la primera y tercera cohorte y al $93 \%$ en la segunda, lo que quiere decir que al final del periodo de estudio entre el $10 \%$ y el $7 \%$ de los estudiantes de estas tres cohortes aún se mantenía en el sistema.

La tasa de azar indica la probabilidad de que un individuo que sobrevive al instante $t$ sobreviva al siguiente intervalo. Esto quiere decir que los estudiantes de la primera cohorte que se mantienen activos al primer año tienen una probabilidad de $20,6 \%$ de mantenerse activos en el siguiente intervalo. En la segunda cohorte la probabilidad aumenta en tres puntos porcentuales al indicar que los que se mantienen activos al primer año tienen una probabilidad de $23,6 \%$ de mantenerse activos al segundo año. En la tercera cohorte la probabilidad es de $25,2 \%$ (cuadro 4).

Al calcular el estadístico Log Rank el resultado muestra un valor de 8,9 y un valor de $p$ de 0,012 , estos valores indican que hay diferencias entre las cohortes al $5 \%$ de significancia. Este estadístico es utilizado para hacer comparaciones entre la sobrevivencia o mortalidad de grupos. Para los tres grupos de estudio establecidos, o sea las cohortes, indica que hay diferencias en el comportamiento de estos para la ocurrencia del evento llamado deserción. 
Cuadro 4. Extracto de la tabla de mortalidad de las tres cohortes estudiadas

\begin{tabular}{|c|c|c|c|c|c|c|}
\hline $\begin{array}{l}\text { Momento } \\
\text { de inicio del } \\
\text { intervalo } \\
\end{array}$ & $\begin{array}{l}\text { Número que } \\
\text { entra en el } \\
\text { intervalo }\end{array}$ & $\begin{array}{l}\text { Número que } \\
\text { sale en el } \\
\text { intervalo }\end{array}$ & $\begin{array}{l}\text { Número de } \\
\text { eventos } \\
\text { terminales } \\
\end{array}$ & $\begin{array}{l}\text { Proporción } \\
\text { que termina }\end{array}$ & $\begin{array}{l}\text { Proporción } \\
\text { acumulada } \\
\text { que sobrevive } \\
\text { al final del } \\
\text { intervalo }\end{array}$ & $\begin{array}{l}\text { Tasa de } \\
\text { impacto }\end{array}$ \\
\hline \multicolumn{7}{|c|}{ Cohorte 1998-1 } \\
\hline $0-2$ & 2782 & 0 & 1313 & 0,472 & 0,528 & 0,206 \\
\hline $3-5$ & 1469 & 21 & 494 & 0,339 & 0,349 & 0,136 \\
\hline $6-8$ & 954 & 78 & 193 & 0,211 & 0,276 & 0,079 \\
\hline $9-11$ & 683 & 67 & 91 & 0,140 & 0,237 & 0,050 \\
\hline $12-14$ & 525 & 81 & 52 & 0,107 & 0,212 & 0,038 \\
\hline $15-17$ & 392 & 56 & 56 & 0,154 & 0,179 & 0,056 \\
\hline $18-20$ & 280 & 41 & 26 & 0,100 & 0,161 & 0,035 \\
\hline $21-23$ & 213 & 31 & 11 & 0,056 & 0,152 & 0,019 \\
\hline $24-26$ & 171 & 30 & 10 & 0,064 & 0,142 & 0,022 \\
\hline $27-29$ & 131 & 27 & 13 & 0,111 & 0,127 & 0,039 \\
\hline $30-32$ & 91 & 15 & 8 & 0,096 & 0,114 & 0,034 \\
\hline $33-35$ & 68 & 17 & 3 & 0,050 & 0,109 & 0,017 \\
\hline $36-38$ & 48 & 13 & 3 & 0,072 & 0,101 & 0,025 \\
\hline $39-41$ & 32 & 11 & 0 & 0,000 & 0,101 & 0,000 \\
\hline $42-44$ & 21 & 21 & 0 & 0,000 & 0,101 & 0,000 \\
\hline \multicolumn{7}{|c|}{ Cohorte 1998-2 } \\
\hline $0-2$ & 2233 & 0 & 1169 & 0,524 & 0,476 & 0,236 \\
\hline $3-5$ & 1064 & 9 & 366 & 0,345 & 0,312 & 0,139 \\
\hline $6-8$ & 689 & 33 & 149 & 0,222 & 0,243 & 0,083 \\
\hline $9-11$ & 507 & 41 & 70 & 0,144 & 0,208 & 0,052 \\
\hline \begin{tabular}{|l|}
$12-14$ \\
\end{tabular} & 396 & 63 & 44 & 0,121 & 0,183 & 0,043 \\
\hline \begin{tabular}{|l|}
$15-17$ \\
\end{tabular} & 289 & 40 & 31 & 0,115 & 0,162 & 0,041 \\
\hline \begin{tabular}{|l|}
$18-20$ \\
\end{tabular} & 218 & 29 & 22 & 0,108 & 0,144 & 0,038 \\
\hline \begin{tabular}{|l}
$21-23$ \\
\end{tabular} & 167 & 34 & 11 & 0,073 & 0,134 & 0,025 \\
\hline $24-26$ & 122 & 16 & 10 & 0,088 & 0,122 & 0,031 \\
\hline $27-29$ & 96 & 19 & 5 & 0,058 & 0,115 & 0,020 \\
\hline
\end{tabular}




\begin{tabular}{|l|l|l|l|l|l|l|}
\hline $30-32$ & 72 & 16 & 4 & 0,063 & 0,108 & 0,022 \\
\hline $33-35$ & 52 & 16 & 3 & 0,068 & 0,100 & 0,024 \\
\hline $36-38$ & 33 & 10 & 4 & 0,143 & 0,086 & 0,051 \\
\hline $39-41$ & 19 & 8 & 3 & 0,200 & 0,069 & 0,074 \\
\hline $42-44$ & 8 & 8 & 0 & 0,000 & 0,069 & 0,000 \\
\hline Cohorte 1998-3 & & & & & \\
\hline $0-2$ & 1641 & 0 & 900 & 0,548 & 0,452 & 0,252 \\
\hline $3-5$ & 741 & 1 & 203 & 0,274 & 0,328 & 0,106 \\
\hline $6-8$ & 537 & 24 & 89 & 0,170 & 0,272 & 0,062 \\
\hline $9-11$ & 424 & 41 & 53 & 0,131 & 0,236 & 0,047 \\
\hline $12-14$ & 330 & 62 & 30 & 0,100 & 0,213 & 0,035 \\
\hline $15-17$ & 238 & 55 & 18 & 0,086 & 0,195 & 0,030 \\
\hline $18-20$ & 165 & 15 & 21 & 0,133 & 0,169 & 0,048 \\
\hline $21-23$ & 129 & 23 & 10 & 0,085 & 0,154 & 0,030 \\
\hline $24-26$ & 96 & 13 & 6 & 0,067 & 0,144 & 0,023 \\
\hline $27-29$ & 77 & 14 & 3 & 0,043 & 0,138 & 0,015 \\
\hline $30-32$ & 60 & 10 & 5 & 0,091 & 0,125 & 0,032 \\
\hline $33-35$ & 45 & 11 & 2 & 0,051 & 0,119 & 0,017 \\
\hline $36-38$ & 32 & 9 & 4 & 0,145 & 0,102 & 0,052 \\
\hline $39-41$ & 19 & 7 & 0 & 0,000 & 0,102 & 0,000 \\
\hline $42-44$ & 12 & 12 & 0 & 0,000 & 0,102 & 0,000 \\
\hline & & & & & & \\
\hline
\end{tabular}

Fuente: elaboración propia

\section{Discusión}

Los textos revisados atribuyen una multiplicidad de factores como detonantes del fenómeno de la deserción a nivel general, situación que se aplica perfectamente a la Universidad Estatal a Distancia, en donde se pueden agrupar en tres grandes factores: los propios del o de la estudiante, de su familia y de la institución.

La disciplina, la organización y el tiempo dedicado a estudiar son aspectos importantes que todo estudiante debe buscar para lograr el éxito académico. No 
obstante, si los estudiantes tienen compromisos familiares o laborales estos dedican menos tiempo a su estudio.

Al estudiar los motivos personales de la deserción en las universidades públicas Brenes (2006) indicó que "casi un 15\% de los estudiantes abandonaron la universidad por aspectos relacionados con su familia como son el matrimonio, embarazo, atención de niños o personas enfermas, así como problemas de salud, emocionales o afectivos» (p.29).

Por otro lado, los aspectos institucionales también tienen su cuota de responsabilidad con relación a la deserción de los y las estudiantes. Kempfer (2001) realizó una revisión de 234 estudios de investigación sobre el tema de la deserción en la educación a distancia y señaló que el proceso de admisión, la calidad de los apoyos de aprendizaje y la interacción entre el tutor y el estudiante son tres elementos importantes para garantizar el éxito o el fracaso del estudiante.

Muchos de ellos llegan a la educación superior con bases académicas deficientes, lo que dificulta su progreso y éxito. Al matricular en un modelo a distancia, muy diferente al que están acostumbrados, los estudiantes se ven en la necesidad de adaptarse al modelo, y no muchos de ellos lo consiguen. Brenes (2006) indicó en su estudio que los desertores de la UNED señalan la metodología a distancia como la opción ideal para sus condiciones particulares, pero que les fue muy difícil su adaptación a ella.

Otro aspecto importantes es que la UNED no realiza examen de admisión, por esta razón, al no existir ese filtro inicial, muchos de los estudiantes conocen sus deficiencias académicas en las primeras asignaturas matriculadas y al reprobarlas se desmotivan y muchos abandonan la carrera. 
La declaración de la deserción como un tema de interés institucional el 7 de junio de 2001, según la resolución del Consejo Universitario N. ${ }^{\circ}$ 1521-2001, marca un punto de arranque de las autoridades universitarias de la UNED por una preocupación constante en el tema.

Muestra de ello, como se comentó anteriormente, es el cambio sufrido en los porcentajes de aprobación de las materias, los cuales pasaron de $59,7 \%$ en el primer cuatrimestre de 2000 a 61,6\% en el tercer cuatrimestre 2012.

Así como existen múltiples factores identificados como causantes de la deserción, también existen a nivel de la UNED múltiples instancias que pueden y están realizando esfuerzos por retener a los muchachos y las muchachas en el sistema de educación superior a distancia.

No obstante, estos esfuerzos deben ir acompañados de recurso humano capacitado y también de recursos tecnológicos. Por tanto, para experimentar cambios significativos en los indicadores de deserción se deben fortalecer prioritariamente aquellas instancias de la universidad que trabajan directamente con los y las estudiantes. Entre ellas se pueden nombrar las pertenecientes a la Dirección de Asuntos Estudiantiles (DAES) de la Vicerrectoría Ejecutiva, ellas son la Oficina de Orientación y Desarrollo y la Oficina de Atención Socioeconómica, instancias de la universidad que ayudan a la retención de los muchachos y las muchachas mediante sus diversos programas.

Este fortalecimiento permitirá un acercamiento de las instancias respectivas dirigida hacia los y las estudiantes. La DAES ha dado algunos pasos en esta línea, el Programa de Orientación y Atención Psicoeducativa ha logrado identificar aquellas asignaturas con mayor grado de repitencia y también ha identificado a los y las estudiantes con mayor riesgo, entre otros logros (UNED, 2013). 
Por su parte, la Oficina de Atención Socioeconómica, además de los programas de becas, también brinda apoyo mediante los programas de Formación Estudiante Becario, Atención Socioeconómica Regional y Apoyo Socioeconómico con Fondos Específicos (UNED, 2013).

Estas oficinas, al igual que otras de apoyo académico, deben ser fortalecidas tanto presupuestariamente como en términos de recurso humano capacitado, para brindar acompañamiento a los y las estudiantes y una adecuada orientación vocacional. La motivación y un contacto permanente con ellos y ellas son factores claves para su retención.

La Vicerrectoría Académica, a través de sus escuelas, debe estar en constante autoevaluación, incorporando técnicas novedosas que favorezcan el aprendizaje a distancia, al igual que el uso de tecnologías de información que faciliten en gran medida el aprendizaje de los y las estudiantes, en especial en aquellas asignaturas con mayor grado de dificultad. En el 2013 se reportaron 115 diseños de asignaturas y 21 rediseños (UNED, 2013), este esfuerzo impacta también los procesos de acreditación en el tema de calidad. Se cuenta en el año 2013 con 13 programas acreditados por SINAES (UNED, 2013).

Otro esfuerzo importante de esta vicerrectoría es la elaboración del Plan Nacional Académico 2012-2017, el cual se construyó pensando en cuatro dimensiones hacia las que se orientarán los esfuerzos de la academia, estas son «mediación pedagógica, evaluar para aprender, territorialidad académica y tecnologías digitales para el aprendizaje» (UNED, 2013, p.31).

Se debe fortalecer la Dirección de Producción Académica con todas sus dependencias: Programa de Aprendizaje en Línea, Programa de Audiovisuales, Programa de Material Didáctico Escrito, Programa de Producción Multimedia y Programa de Videoconferencias. Cada una de ellas con sus importantes productos 
orientados a hacer eficiente la mediación pedagógica, entre ellos están las tutorías, los multimedios y los videos, así como las unidades didácticas y los libros, entre otros, y por último, sin ser menos importante, se encuentra la administración de plataformas de aprendizaje.

Un ingrediente adicional que no puede dejar de mencionarse es la articulación entre todas estas instancias. Este es un reto al que se enfrenta el Rector y los Vicerrectores de la universidad en su periodo de gestión.

Por otro lado, la universidad debe propiciar espacios de socialización donde los y las estudiantes se conozcan, sepan quiénes son sus compañeros y compañeras de grupo y puedan construir conocimiento en conjunto.

Una forma utilizada actualmente son los espacios conocidos como foros sociales, que son herramientas de las plataformas de aprendizaje del Programa de Aprendizaje en Línea. Los y las estudiantes, una vez que se presentan a través de este espacio, logran establecer otros espacios de comunicación más cercana como los grupos de WhatsApp y Facebook, entre otros. Estos espacios de comunicación y socialización les permiten evacuar dudas entre ellos y asimilar la materia de una mejor forma. Aunque no es comparable con la camaradería entre compañeros de un sistema presencial, esta comunicación les permite una identificación con los compañeros y las compañeras de grupo.

De igual manera, es importante facilitar el acceso a internet a nivel de los centros universitarios mediante la instalación de banda ancha y computadoras en buen estado y actualizadas, ya que el acceso a internet en algunas zonas alejadas es deficiente. Por otro lado, las plataformas de aprendizaje, como el Moodle, deben ser fáciles de trabajar en dispositivos móviles como celulares inteligentes, ya que cada vez son más los y las estudiantes que utilizan estos equipos en cualquier lugar y hora. 
Los mayores esfuerzos para lograr que el o la estudiante de primer ingreso se mantenga en el sistema se deben realizar en los primeros tres cuatrimestres de su vida estudiantil, ya que es en este periodo en el que se dan los porcentajes altos de deserción. Tan solo en el año 2013 ingresaron 10347 estudiantes de primer ingreso a esta institución (UNED, 2013).

No hay que olvidar también que el éxito académico (graduación) de los y las estudiantes es otro momento crítico en la vida académica de los muchachos y las muchachas que debe atenderse con prioridad. Sin embargo, este tema no es sujeto de análisis en este trabajo.

\section{Conclusiones}

Los resultados del análisis de datos en este estudio no son muy diferentes de los encontrados en otros estudios, estos muestran que las cifras de deserción en los primeros tres años son altas y sugieren una atención importante por parte de las autoridades universitarias.

La universidad debe replantearse la definición de deserción utilizada en el Reglamento General Estudiantil, ya que tal como está definida incluye en una sola cifra a los desertores de los programas y a los desertores de la institución.

Se deben realizar estudios periódicos de seguimiento de los desertores, que utilicen una metodología similar y que sirvan de insumo para la toma de decisiones de las autoridades.

Se deben fortalecer los sistemas institucionales de información, por ejemplo el Sistema de Administración de Estudiantes (SAE), para que almacene información oportuna y confiable que facilite el estudio de este tema. 
Las estadísticas de rendimiento académico deben ser utilizadas con mayor frecuencia para realizar monitoreo y evaluación de las asignaturas con porcentajes altos de retiro y/o repitencia.

Son pocos los estudiantes que logran terminar con éxito sus estudios. Del total de estudiantes que ingresaron en 1998 tan solo un 16,89\% había obtenido un título universitario en el año 2012. Esta cifra debe ser valorada por las autoridades universitarias con el propósito de buscar aquellas causas que provocan un porcentaje tan bajo.

La institución debe enfocar sus esfuerzos en dar un buen acompañamiento a los y las estudiantes en los primeros tres periodos académicos después de su ingreso. Tan solo en los primeros dos cuatrimestres después de la primera matrícula se retiraron alrededor del $50 \%$ de los y las estudiantes en las tres cohortes estudiadas. Sobre todo porque es baja la probabilidad de estudiantes (entre el $20 \%$ y $25 \%$ ) que lograron llegar al tercer cuatrimestre y siguieron estudiando.

Los resultados de la tabla de mortalidad indican que las tres cohortes estudiadas son diferentes, según el estadístico Log Rank al 5\% de significancia. Tomando en consideración este resultado la universidad debe realizar investigaciones para conocer las características de los y las estudiantes de primer ingreso de los tres periodos académicos, para orientar sus apoyos de acuerdo a estas características. 


\section{Referencias}

Barrientos, Z. y Umaña, R. (2009). Deserción estudiantil en posgrados semipresenciales de la Universidad Estatal a Distancia (UNED), Costa Rica: ¿Deserción o retraso? Cuadernos de investigación UNED, 1(2), 141-149. Recuperado http://investiga.uned.ac.cr/revistas/index.php/cuadernos/article/view/227

Brenes, I. (2005). Deserción y repitencia en la educación superior universitaria de Costa Rica. Consejo Nacional de Rectores, San José, Costa Rica.

Brenes, I. (2006). Motivos e implicancias de la deserción en la cohorte de admitidos en 1996 a las instituciones de educación superior universitaria estatal. Consejo Nacional de Rectores, San José, Costa Rica.

Castillo, M. (2008). Tasas de deserción en la Universidad Estatal a Distancia de Costa Rica. Actualidades Investigativas en Educación, 8(1), 1-32. Recuperado de http://revista.inie.ucr.ac.cr/ediciones/controlador/Article/accion/show/articulo/ tasas-de-desercion-en-la-universidad-estatal-a-distancia-de-costa-rica.html

Chaves, E. (2000). Deserción y rendimiento académico en la UNED: 1996-1999. Documento CIEI 011-2000. Universidad Estatal a Distancia, San José, Costa Rica.

Chávez, F. J., Panchi, A. y Montoya, S. (2007). Abandono de Estudios en la Educación Superior a Distancia. Un análisis de caso. Innovación Educativa, 7(39), 5-17. Recuperado de http://www.redalyc.org/pdf/1794/179421210002.pdf

CONARE-OPES (s.f.). Informe Nacional sobre la Educación Superior: Costa Rica. Recuperado de http://www.hacienda.go.cr/centro/datos/Articulo/Informe\%20Nacional\%20so bre\%20la\%20educaci\%C3\%B3n\%20superior-CR.pdf

Estado de la Nación (2013). Cuarto Informe Estado de la Educación. San José, Costa Rica.

Himmel, E. (2002). Modelos de análisis de la deserción estudiantil en la educación superior. Calidad de la Educación: retención y movilidad estudiantil en la educación superior, 17, 91-107. Recuperado de

http://www.cned.cl/public/secciones/seccionpublicaciones/doc/35/cse_articul 0141.pdf 
Kempfer, H. (2001). Cómo reducir la deserción en la educación a distancia. Universidad Estatal a Distancia, San José, Costa Rica.

Ministerio de Educación Nacional (2009). Deserción estudiantil en la educación superior colombiana, metodología de seguimiento, diagnóstico y elementos para su prevención. Colombia: Imprenta Nacional de Colombia.

Montes, I., Almonacid, P., Gómez, S., Zuluaga, F. y Tamayo, E. (2010). Análisis de la deserción estudiantil en los programas de pregrado de la universidad EAFIT. Documentos de trabajo Economía y Finanzas. Centro de Investigaciones Económicas y Financieras. Recuperado de https://repository.eafit.edu.co/bitstream/handle/10784/912/2010_10_Isabel_ Montes.pdf?sequence $=1$

Osorio, A. y Jaramillo, C. (1999). Deserción estudiantil en los Programas de Pregrado 1995-1998. Ministerio de Educación Nacional. Medellín, Colombia: Imprenta Nacional de Colombia

Umaña, R. (2004). Elaboración de una propuesta contextual, teórica y metodológica para el abordaje de la deserción de estudiantes en la Universidad Estatal a Distancia de Costa Rica. Universidad Estatal a Distancia, San José, Costa Rica.

Umaña, R. (2011). Factores relacionados con la deserción de estudiantes de grado en la Universidad Estatal a Distancia. Cohorte de estudio: año 2000 primer cuatrimestre. Informe final de la etapa II de investigación. Universidad Estatal a Distancia, San José, Costa Rica.

UNED (2012a). Anuario estadístico. Centro de Investigación y Evaluación Institucional, Universidad Estatal a Distancia. San José, Costa Rica.

UNED (2012b). Reglamento General Estudiantil. Universidad Estatal a Distancia, San José, Costa Rica.

UNED (2013). Informe de labores. Recuperado de http://www.uned.ac.cr/transparencia/informe-de-labores-2013 